\title{
Genetic and Environmental Contributions to Perceived Intensity and Pleasantness of Androstenone Odor: An International Twin Study
}

\author{
Antti Knaapila • Hely Tuorila • Karri Silventoinen • \\ Margaret J. Wright • Kirsten O. Kyvik • \\ Lynn F. Cherkas • Kaisu Keskitalo • Jonathan Hansen • \\ Nicholas G. Martin • Tim D. Spector • Jaakko Kaprio • \\ Markus Perola
}

Received: 3 September 2007 / Accepted: 3 December 2007 / Published online: 3 January 2008

(C) 2008 Springer Science + Business Media, LLC

\begin{abstract}
We estimated the genetic and environmental components of variation in perceived intensity and pleasantness of androstenone, an odorous compound showing specific anosmia, by modeling twin data from Finland, Denmark, the UK, and Australia. The pooled data comprised 917 twin individuals (338 are male and 579 are female; aged from 10 to 83years) including 126 complete monozygous and 264 dizygous twin pairs as well as 137
\end{abstract}

A. Knaapila $(\bowtie) \cdot H$. Tuorila $\cdot$ K. Keskitalo

Department of Food Technology, University of Helsinki,

P.O. Box 66, 00014 Helsinki, Finland

e-mail: antti.knaapila@helsinki.fi

\author{
A. Knaapila $\cdot$ K. Keskitalo $\cdot$ M. Perola \\ Department of Molecular Medicine, \\ National Public Health Institute, \\ P.O. Box 104, 00251 Helsinki, Finland

\section{K. Silventoinen $\cdot$ J. Kaprio} \\ Department of Public Health, University of Helsinki, \\ P.O. Box 41, 00014 Helsinki, Finland
}

M. J. Wright $\cdot$ J. Hansen • N. G. Martin

Queensland Institute of Medical Research,

P.O. Royal Brisbane Hospital,

Brisbane, Queensland 4029, Australia

\section{K. O. Kyvik}

Institute of Regional Health Research and the Danish Twin

Registry, Institute of Public Health,

University of Southern Denmark,

J.B. Winsløws Vej 19,

5000 Odense C, Denmark twin individuals without their co-twin. They rated intensity and pleasantness of androstenone and citronellal (control) odors using nine categories. Additive genetic effects (heritability) contributed 28 and $21 \%$ to the variation in the perceived intensity and pleasantness of androstenone, respectively, but negligibly to variations in citronellal perception. A strong genetic correlation existed between the intensity and pleasantness of androstenone, whereas the

\author{
L. F. Cherkas $\cdot$ T. D. Spector \\ Twin Research and Genetic Epidemiology Unit, \\ King's College London, \\ Lambeth Palace Road, \\ London SE1 7EH, UK \\ J. Kaprio \\ Department of Mental Health and Alcohol Research, \\ National Public Health Institute, \\ Mannerheimintie 166, \\ 00300 Helsinki, Finland \\ M. Perola \\ Department of Medical Genetics, University of Helsinki, \\ P.O. Box 63, 00014 Helsinki, Finland
}


environmental correlation was negligible. These results suggest that both intensity and pleasantness of androstenone are moderately influenced by genetic factors and that the traits are modified by an overlapping set of genes.

Keywords Heritability · Olfaction · Psychophysics · Scratch-and-sniff $\cdot$ Smell $\cdot$ Specific Anosmia

\section{Introduction}

Androstenone (5- $\alpha$-androst-16-en-3-one) is an odorous steroid that shows specific anosmia: Some individuals with otherwise normal olfactory acuity are unable to perceive its odor. Estimates for the prevalence of androstenone anosmia range from 2 to $75 \%$, but from 20 to $40 \%$ in most studies (for a review, see Bremner et al. 2003). Boyle et al. (2006) demonstrated that many androstenone anosmics were able to detect androstenone at high concentrations based solely on trigeminal activation and suggested that this explains the variation in estimates of androstenone anosmia prevalence. The view of the dichotomous perception of androstenone originates from a bimodal distribution of detection thresholds (Labows and Wysocki 1984). However, the trait can also be regarded as continuous, as quantitative variation exists in the thresholds. Among androstenone smellers, variation in the pleasantness of androstenone odor is also observed: Some individuals describe the odor as stale urine, whereas others use descriptors like sweet, musky, and woody (Labows and Wysocki 1984).

Age, sex, prior exposure, and genetic factors influence androstenone perception. Although some adults are anosmic to androstenone, most children are able to smell it (Dorries et al. 1989; Wysocki and Gilbert 1989). Among adults, androstenone anosmia is more frequent among men than women (Wysocki and Gilbert 1989; Pierce et al. 2004), although this has not been found in some studies (Wysocki and Beauchamp 1984; Boyle et al. 2006). Repeated exposures seem to sensitize some individuals to androstenone. In a study by Wysocki et al. (1989), half of the initially ostensibly androstenone anosmic subjects, including both male and female subjects, acquired the ability to perceive the odor. However, the sensitization through repeated exposures may be a more general phenomenon. Dalton et al. (2002) demonstrated the sensitization to benzaldehyde and citralva, but only in women at reproductive age. In a recent study, Boulkroune et al. (2007) showed that repetitive exposure to androstadienone (4,16-androstadien-3-one), a steroid chemically related to androstenone, decreased detection thresholds of the odor in males and females.

Genetic control for the ability to detect androstenone has been studied in three small twin studies, which compared intraclass correlations of monozygous (MZ) and dizygous (DZ) twins for detection thresholds of androstenone. Intraclass correlations of MZ twins were higher than those of DZ twins in studies by Wysocki and Beauchamp (1984; 17 MZ and 21 DZ pairs), Gross-Isseroff et al. (1992; $17 \mathrm{MZ}$ and $15 \mathrm{DZ}$ pairs), and Pause et al. (1998; $15 \mathrm{MZ}$ and $15 \mathrm{DZ}$ pairs), indicating that androstenone perception is in part genetically determined. However, no estimate for the relative contribution of genetic effects has been reported to date. In addition, heritability estimate for hedonic response to androstenone is lacking, although there is evidence that pleasantness of some odors shows heritable variation (Knaapila et al. 2007). As individuals highly sensitive to androstenone tend to describe it as more offensive than less sensitive subjects (Labows and Wysocki 1984), we hypothesized that related genetic factors may influence both the perceived intensity and pleasantness of androstenone.

In this paper, we quantified the genetic and environmental components of variation in the perceived intensity and pleasantness of androstenone odor using structural equation modeling of large international twin data including 390 complete twin pairs. We also studied possible common underlying factors determining genetic and environmental correlations between the traits.

\section{Materials and Methods}

\section{Subjects}

A total of 917 twin individuals, including 126 complete monozygous (MZ) and 264 complete dizygous (DZ) twin pairs as well as 137 twin individuals without their co-twin, participated in the study. Only complete twin pairs are genetically informative, but also information from individuals without their co-twin were retained in the data because all individuals contribute to power in basic statistical analyses. Characteristics of the subsamples and the pooled sample are shown in Table 1. Subsamples of the data were from the Finnish Twin Cohort study (FinnTwin12 Cohort; Kaprio et al. 2002; Kaprio 2006), the Danish Twin Registry (Skytthe et al. 2002; Skytthe et al. 2006), the UK Adult Twin Registry (Spector and Williams 2006), and the Brisbane Adolescent Twin Study (Wright and Martin 2004). The ethical principles applied in the present study were approved by the appropriate Ethical Committees and Data Protection Agencies in the respective countries. The zygosities of the twins were determined using DNA-based microsatellite markers (Danish and Australian twins) or questionnaire and/or genotyping (Finnish and British twins). 
Table 1 Characteristics of study samples

\begin{tabular}{|c|c|c|c|c|c|c|c|c|c|c|}
\hline & \multicolumn{8}{|l|}{ Subsample } & \multicolumn{2}{|c|}{ Pooled sample } \\
\hline & \multicolumn{2}{|l|}{ Finland } & \multicolumn{2}{|l|}{ Denmark } & \multicolumn{2}{|l|}{ UK } & \multicolumn{2}{|l|}{ Australia } & \multirow[b]{2}{*}{ Number } & \multirow[b]{2}{*}{ Percent } \\
\hline & Number & Percent & Number & Percent & Number & Percent & Number & Percent & & \\
\hline \multicolumn{11}{|l|}{ Individuals } \\
\hline Males & 81 & 37 & 158 & 47 & 7 & 4 & 92 & 46 & 338 & 37 \\
\hline Females & 138 & 63 & 178 & 53 & 156 & 96 & 107 & 54 & 579 & 63 \\
\hline Total & 219 & 100 & 336 & 100 & 163 & 100 & 199 & 100 & 917 & 100 \\
\hline \multicolumn{11}{|c|}{ Complete pairs $^{\mathrm{a}}$} \\
\hline $\mathrm{MZ}$ & 47 & 49 & 0 & 0 & 60 & 75 & 19 & 19 & 126 & 32 \\
\hline $\mathrm{DZ}$ & 48 & 51 & 116 & 100 & 20 & 25 & 80 & 81 & 264 & 68 \\
\hline Total & 95 & 100 & 116 & 100 & 80 & 100 & 99 & 100 & 390 & 100 \\
\hline \multicolumn{11}{|l|}{ Age (years) } \\
\hline Range & $22-23$ & & $24-60$ & & $19-83$ & & $10-17$ & & $10-83$ & \\
\hline Mean (SD) & $22.6(0.5)$ & & $44.9(9.3)$ & & $54.9(12.2)$ & & $14.3(1.9)$ & & $34.7(17.2)$ & \\
\hline
\end{tabular}

${ }^{\mathrm{a}}$ In addition to complete pairs, data included 137 twin individuals without their co-twin.

\section{Procedure}

Scratch-and-sniff stimuli for androstenone (5- $\alpha$-androst- $16-$ en-3-one, CAS no. 18339-16-7) and citronellal (3,7dimethyl-6-octenal, CAS no. 106-23-0, lemon odor) were presented among a set also containing isovaleric acid (sweaty odor), cinnamaldehyde (cinnamon odor), turpentine, chocolate aroma, and blank. In addition to androstenone, citronellal was included in the present study as a clearly detectable control odorant with no known specific anosmia, whereas the results of the other odors are not reported in this paper. Androstenone was supplied by Sigma-Aldrich (Cat no. A8008) and all other odors by Quest International (presently Givaudan). The odor samples were marked with three-digit random codes and presented in individually randomized orders to the subjects. Only androstenone, citronellal, isovaleric acid, and cinnamaldehyde were presented in the UK. The odor stimuli were chemically identical across the different subsamples. With the exception of the UK subsample, two stimuli for training (odorless and vanillin) were presented before the stimuli being rated. Each odorant was microencapsulated in a label $(1.9 \times 6.5 \mathrm{~cm})$ on a separate sheet $(14.8 \times 21.1 \mathrm{~cm})$, which also contained the rating scales and brief instructions in appropriate language (Finnish, Danish, or English). In the labels, androstenone was present as $0.1 \%$ solution in diethyl phthalate and citronellal as pure (purity 92.5\%). The sheets containing the scratch-and-sniff labels were manufactured by Kent Art Printers (Kent, UK).

The subjects' task was to scratch the label using the tip of a pencil to release the odor, sniff the label immediately after scratching, and rate the intensity and pleasantness of the odor using a nine-point category scale. Endpoints of the scales were "No odor" (rating 1) and "Extremely strong odor" (rating 9) for the intensity scale and "Extremely unpleasant" (rating 1) and "Extremely pleasant" (rating 9) for the pleasantness scale, that was also anchored with "Neither pleasant nor unpleasant" (rating 5) in the middle. The pleasantness was rated first. After rating the pleasantness and intensity of an odor, subjects evaluated the identity of the odor by choosing the best descriptor for the odor from 13 alternatives (no odor, rose, lemon, vanilla, chocolate, cinnamon, onion, malt, smoke, turpentine, sweat, urine, or another odor (with space for free description)). Reliability of the odor ratings, described with test-retest correlation (Pearson's $r, n=26$, unpublished results), was 0.84 and 0.80 for intensity of androstenone and citronellal, respectively, and 0.56 and 0.81 for pleasantness of androstenone and citronellal, respectively.

The data were collected at twin research units in Finland (Helsinki) and Australia (Brisbane). In the UK, the data were collected from volunteers at the Twin Party (July 2006, London). In Denmark, the odor stimuli were mailed to twins, who rated the stimuli at home and returned the data by mail. In addition to the brief instructions for odor evaluations in the odor stimuli sheets, the subjects were given detailed oral (Finland, UK, Australia) or written (Denmark) instructions for the task. The participants also completed a background information questionnaire and gave a written informed consent.

\section{Statistical Analysis}

Ratings of odors were regarded as continuous variables. If the intensity of an odor was rated as "No odor" (rating 1), the pleasantness rating of the odor was omitted from the analyses. Effects of country and sex (fixed factors) on ratings were explored using two-way analysis of variance 
including age as a covariate. The effect of sampling of twins based on twin pairs was taken into account by including twin pair number as a random effects variable in the model.

The phenotypic variance in ratings was decomposed into genetic and environmental components using structural equation modeling of the raw twin data. Modeling of variance components were performed using the program Mx, version 1.5 (Neale et al. 2003). As the ratings were influenced by age, sex, and country of subsample (see "Results"), they were included as covariates in the modeling.

The estimation of genetic and environmental variance components is based on the assumption that MZ twins share $100 \%$ and DZ twins, on average, $50 \%$ of their segregating genes, whereas the common environment is assumed to affect the MZ and DZ twins equally. The phenotypic variance can be decomposed into components of additive genetic effects (A), non-shared (individual) environmental effects (E), and either non-additive genetic effects (D) or shared (common) environmental effects (C), if only twins reared together are involved. In this case, components $\mathrm{C}$ and $\mathrm{D}$ cannot be modeled simultaneously due to the confounding nature of these components: Component $\mathrm{C}$ reduces the differences between $\mathrm{MZ}$ and DZ twin correlations, whereas component D increase this distance. The variation produced by various sources of error is by definition included in the $\mathrm{E}$ component. As the comparison of intraclass correlations of the MZ and DZ twins implied the existence of non-additive genetic effects (i.e., the DZ correlations were less than half of the MZ correlations, Table 2), the model including components A, $\mathrm{D}$, and $\mathrm{E}$ was chosen as the starting point for analyses. Submodels of the ADE model were then tested to seek a more parsimonious model and against the saturated model for the validity of the assumptions of twin modeling (equal

Table 2 Intraclass correlations among monozygous (MZ) and dizygous (DZ) twins for the intensity and pleasantness ratings in the pooled data

\begin{tabular}{lllll}
\hline \multicolumn{5}{c}{ Intraclass correlations } \\
\cline { 2 - 5 } & $\mathrm{r}(\mathrm{MZ})$ & $(95 \% \mathrm{CI})$ & $\mathrm{r}(\mathrm{DZ})$ & $(95 \% \mathrm{CI})$ \\
\hline $\begin{array}{l}\text { Intensity } \\
\text { Androstenone }\end{array}$ & 0.30 & $(0.13 \ldots 0.46)$ & 0.10 & $(-0.02 \ldots 0.22)$ \\
$\begin{array}{l}\text { Citronellal } \\
\text { Pleasantness }\end{array}$ & 0.17 & $(-0.01 \ldots 0.34)$ & 0.03 & $(-0.09 \ldots 0.16)$ \\
Androstenone & 0.25 & $(0.06 \ldots 0.43)$ & 0.07 & $(-0.08 \ldots 0.21)$ \\
Citronellal & -0.05 & $(-0.23 \ldots 0.12)$ & 0.07 & $(-0.06 \ldots 0.19)$ \\
\hline
\end{tabular}

a Pleasantness rating was omitted if the intensity of corresponding odor was rated as "No odor" (rating 1).

95\% CI 95\% Confidence interval means and variances between the MZ and DZ twins and likewise for the twins within a pair; Neale and Cardon 1992). Tests of nested models were based on the change in $\chi^{2}$-statistics compared with the change of degrees of freedom; a statistically significant change in the $\chi^{2}$-statistic indicates that the more parsimonious model does not fit the data well and important parameters are omitted (Table 3).

To explore genetic and environmental correlations between the perceived intensity and pleasantness ratings, we performed a bivariate Cholesky decomposition of variance, which allows one to compute correlations between genetic and environmental variance components affecting these two traits and thus explaining the observed phenotypic correlation. Significance of correlations was tested by comparing nested models as described above.

\section{Results}

The rating of "No odor" was given to androstenone stimulus by 1 in $5(18.8 \%)$ subjects $(21.9 \%$ of male and $17.6 \%$ of female subjects) and to citronellal stimulus only by 1 in $250(0.4 \%)$ subjects $(0.3 \%$ of male and $0.5 \%$ of female subjects; Fig. 1). This suggested that, in our sample, the occurrence of specific anosmia to androstenone was at an expected level, and general or specific anosmia to citronellal was negligible. As expected (Labows and Wysocki 1984), the more intense the androstenone was experienced, the more often it was evaluated with the descriptor "sweat" or "urine" (Fig. 2).

The mean intensity and pleasantness ratings in the subsamples from different countries and the pooled sample are shown in Fig. 3. Two-way analysis of variance including age as a covariate was used to determine main effects of country and sex and their interactions on the ratings. Interaction of country $\times$ sex was significant only for the intensity of citronellal $(p=0.035)$ and was omitted from analysis of other traits. There was a significant main effect of country on both intensity $[F(3,519)=14.87, p<0.001]$ and pleasantness of androstenone $[F(3,476)=8.85, p<0.001]$, as well as on both intensity $[F(3,521)=5.93, p<0.001]$ and pleasantness of citronellal $[F(3,519)=11.41, p<0.001]$. Female subjects rated androstenone as more intense than male subjects did [main effect of sex, $F(1,887)=6.5, p=$ 0.011].

Age did not correlate with perceived intensity or pleasantness of androstenone. However, subjects aged under 16 years rated androstenone, but not citronellal, as more intense than subjects aged 16 years or more $(t=-4.1, p<$ 0.001 ). In the case of citronellal, there was a weak correlation between age and intensity rating $[-0.13(95 \%$ confidence interval (CI) $-0.20 \ldots-0.07), p<0.001]$ and between age and pleasantness rating [0.23 (95\% CI $0.17 \ldots 0.29), p<0.001]$. 
Table 3 Model fit statistics for univariate models for intensity and pleasantness of androstenone and citronellal odors

\begin{tabular}{|c|c|c|c|c|}
\hline & \multicolumn{2}{|l|}{ Intensity } & \multicolumn{2}{|l|}{ Pleasantness } \\
\hline & Androstenone & Citronellal & Androstenone & Citronellal \\
\hline \multicolumn{5}{|l|}{ Saturated model } \\
\hline$\chi^{2}$ & 3435.616 & 2941.900 & 2598.973 & 3085.759 \\
\hline$d f$ & 748 & 755 & 619 & 754 \\
\hline \multicolumn{5}{|l|}{ ADE model } \\
\hline$\chi^{2}$ & 3438.023 & 2943.252 & 2600.753 & 3088.521 \\
\hline$d f$ & 754 & 761 & 625 & 760 \\
\hline \multicolumn{5}{|l|}{ AE model } \\
\hline$\chi^{2}$ & 3438.812 & 2943.613 & 2601.066 & 3088.521 \\
\hline$d f$ & 755 & 762 & 626 & 761 \\
\hline$\Delta \chi^{2}$ compared to ADE model & 0.789 & 0.361 & 0.313 & 0.000 \\
\hline$\Delta d f$ compared to ADE model & 1 & 1 & 1 & 1 \\
\hline$p$ value & 0.374 & 0.548 & 0.576 & 1.000 \\
\hline$\Delta \chi^{2}$ compared to saturated model & 3.196 & 1.713 & 2.093 & 2.762 \\
\hline$\Delta d f$ compared to saturated model & 7 & 7 & 7 & 7 \\
\hline$p$ value & 0.866 & 0.974 & 0.955 & 0.906 \\
\hline \multicolumn{5}{|l|}{ E model } \\
\hline$\chi^{2}$ & 3451.991 & 2947.077 & 2607.383 & 3088.534 \\
\hline$d f$ & 756 & 763 & 627 & 762 \\
\hline$\Delta \chi^{2}$ compared to ADE model & 13.968 & 3.825 & 6.630 & 0.013 \\
\hline$\Delta d f$ compared to ADE model & 2 & 2 & 2 & 2 \\
\hline$p$ value & 0.001 & 0.148 & 0.036 & 0.994 \\
\hline$\Delta \chi^{2}$ compared to saturated model & 16.375 & 5.177 & 8.41 & 2.775 \\
\hline$\Delta d f$ compared to saturated model & 8 & 8 & 8 & 8 \\
\hline$p$ value & 0.037 & 0.739 & 0.394 & 0.948 \\
\hline
\end{tabular}

$A D E$ model Model including additive genetic (A), non-additive genetic (D), and non-shared environmental (E) components; $A E$ model model including components A and E; E model model including component $\mathrm{E}$

Pleasantness and intensity ratings were moderately correlated in the case of androstenone $[-0.27(95 \%$ CI $-0.33 \ldots-0.20)$, $p<0.001$ ], but negligibly in the case of citronellal [0.08 $(95 \%$ CI $0.01 \ldots 0.14), p<0.05]$.

We decomposed the phenotypic variation in odor ratings into genetic and environmental components using structural equation modeling. The data from four countries were pooled to maximize statistical power. In the pooled data, for ratings of intensity of androstenone and citronellal and pleasantness of androstenone, the intraclass correlations of $\mathrm{MZ}$ pairs were more than twice as high than those of DZ pairs (Table 2). This suggested that non-additive genetic effects were involved and that shared (common) environmental effects were unimportant. Thus, we fitted a univariate model including additive genetic (A), nonadditive genetic (D), and non-shared (individual) environmental components (E) to the pooled data first and then searched for a more parsimonious model.

In the case of intensity and pleasantness of androstenone, the goodness-of-fit of the model did not decrease significantly if the component D was omitted from the model. In contrast, omitting component A would have impaired the fit significantly (Table 3 ). Thus, the best univariate model for androstenone was the AE model implying that a significant additive genetic component was involved. The parameter estimates for variance components were computed using this model (Fig. 4). However, for both the intensity and pleasantness of citronellal, the goodness-of-fit did not decrease significantly if both genetic components (A and D) were omitted from the model (Table 3). This indicated that the E model was the most parsimonious model that fitted the data and, thus, that all variance was due to nonfamilial effects, i.e., non-shared environmental effects and error variation. The assumptions of twin modeling (see "Materials and Methods") were not violated when using the final univariate models (AE model for androstenone and $\mathrm{E}$ model for citronellal), as these models did not fit the data significantly worse than the saturated model, which does not make these assumptions (Table 3).

As the genetic component of variance was detected for both intensity and pleasantness of androstenone, a bivariate Cholesky decomposition was performed to determine possible common genetic effects underlying the phenotypic correlation between the traits. The bivariate decomposition including components $\mathrm{A}$ and $\mathrm{E}$ revealed a strong (additive) genetic correlation between intensity and pleasantness 


\section{a}

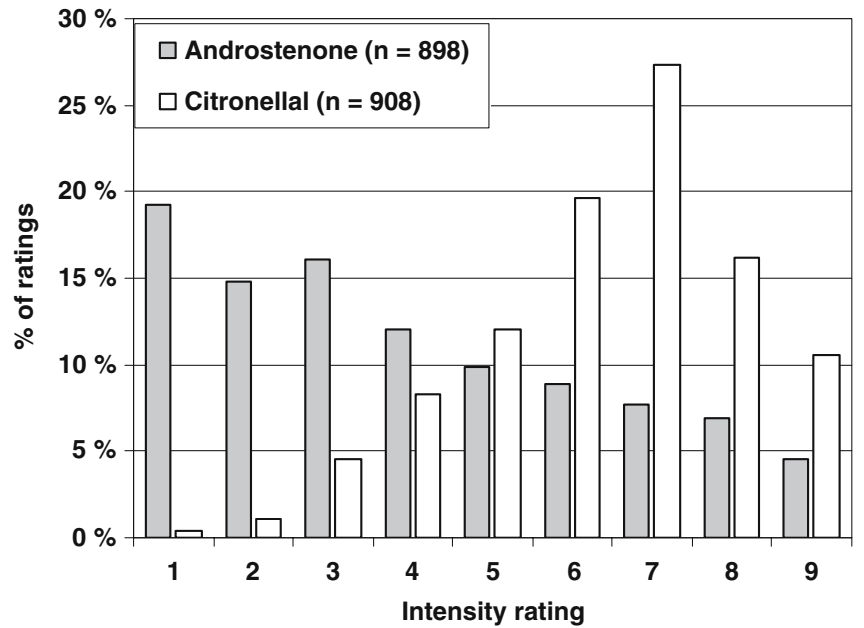

Fig. 1 Distributions of intensity (a) and pleasantness (b) ratings for androstenone and citronellal. The intensity scale were anchored with "No odor" (rating 1) and "Extremely strong odor" (rating 9) and the pleasantness scale with "Extremely unpleasant" (rating 1), "Neither

(Fig. 5), whereas a (non-shared) environmental correlation between them was insignificant and omitted from the model $\left(\Delta \chi^{2}=1.24, \Delta d f=1, p=0.265\right)$. This indicates that the genetic correlation alone can explain the phenotypic correlation between the intensity and pleasantness of androstenone. The bivariate decomposition (Fig. 5) yielded similar parameter estimates for the variance components than the univariate model (Fig. 4).

\section{Discussion}

We estimated the relative contribution of the genetic effects to variation in perceived intensity and pleasantness of

\section{b}

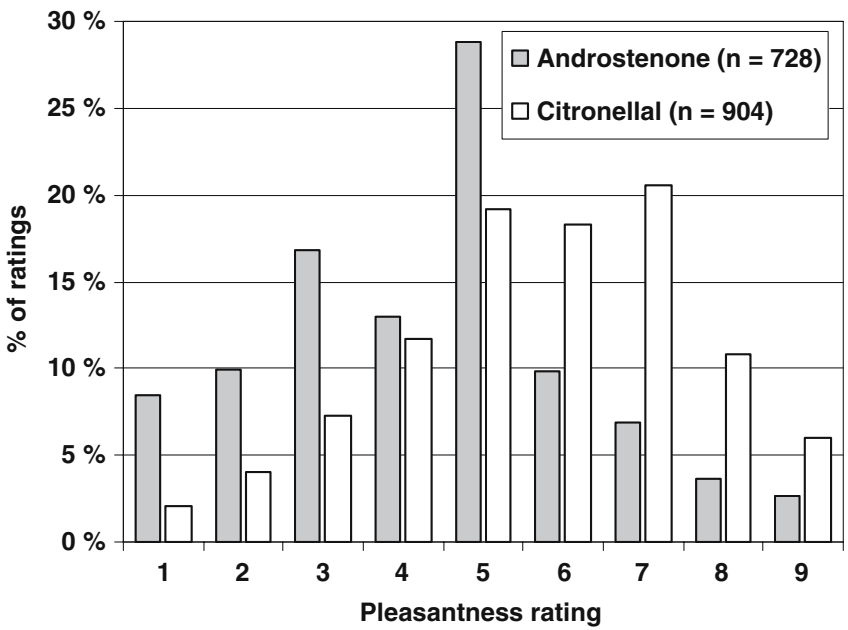

pleasant nor unpleasant" (rating 5), and "Extremely pleasant" (rating 9). Pleasantness ratings of subjects who rated the intensity of the stimulus as "No odor," were omitted

androstenone odor. Evidence for heritability of androstenone anosmia has been found in earlier studies (Wysocki and Beauchamp 1984; Gross-Isseroff et al. 1992; Pause et al. 1998). Recently, Keller et al. (2007) demonstrated that individuals who were homozygous for an allele of odorant receptor OR7D4 gene experienced the odor of androstenone as more intense and more unpleasant than other subjects. However, the magnitude of the genetic effects on subjective perceptions at a population level has not been quantified to date. The earlier twin studies (Wysocki and Beauchamp 1984; Gross-Isseroff et al. 1992; Pause et al. 1998) were based on comparison of intraclass correlations of a relatively small number of twins (30-38 pairs), whereas we applied structural equation modeling to a large international twin
Fig. 2 Proportions of identity evaluations given to androstenone odor relative to its perceived intensity. Identity evaluation was omitted if the intensity of the odor was rated as "No odor" (rating 1)

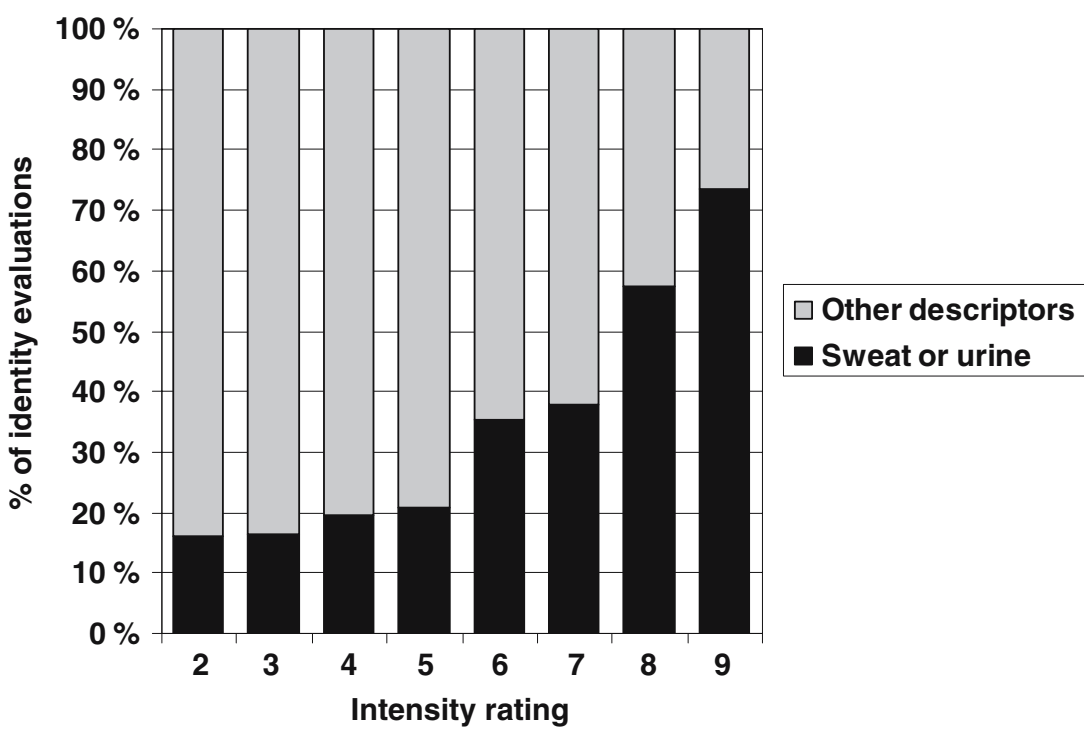




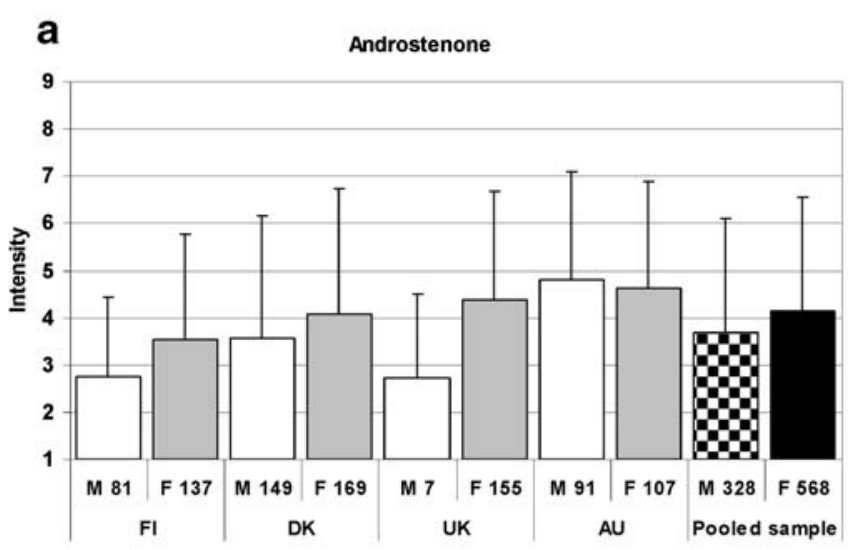

C

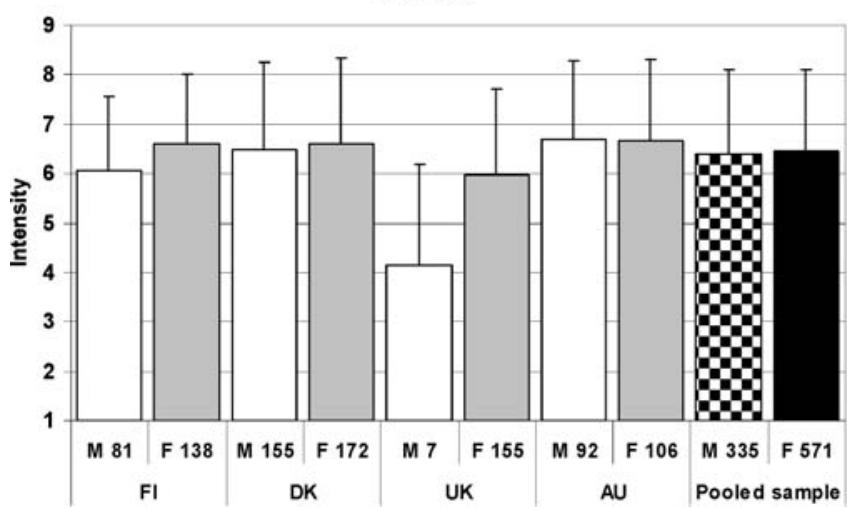

Fig. 3 Mean (error bar indicates standard deviation) intensity and pleasantness ratings of androstenone (a, b) and citronellal (c, d) by male and female subjects ( $M$ and $F$ with number of cases, respectively) in the subsamples from different countries (FI Finland; $D K$ Denmark; $U K$ United Kingdom; $A U$ Australia) and in the pooled

dataset, including 390 twin pairs. To maximize the statistical power for detecting the genetic effects, we pooled our subsamples from different countries. To ensure the validity of the pooling, we included the origin of the subsample and sex and age as covariates in modeling.

The modeling revealed moderate additive genetic components (heritability) for both perceived intensity and pleasantness of androstenone, but most of the variation was due to non-shared (individual) environmental effects. A relatively large measurement error, that by definition is included in the non-shared environmental component, is inevitably present in self-reported ratings of odor stimulus, thus increasing the estimate of the non-shared environmental component and hindering the detection of the genetic component. We cannot rule out variation in temperature in that the odor stimuli were rated, and thus, this variation may have contributed to random and systematic error in results. The results of the androstenone pleasantness should be interpreted cautiously because the test-retest reliability was low. Our data do not provide evidence for shared

\section{b}

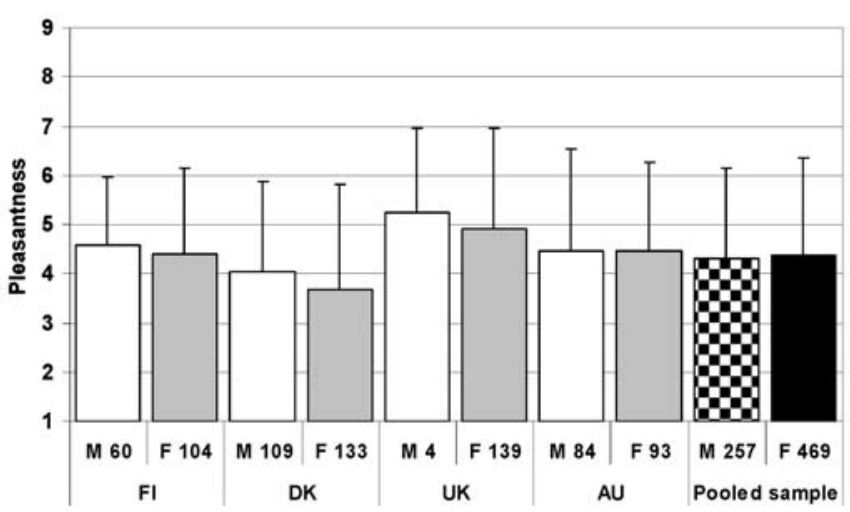

d

Citronellal

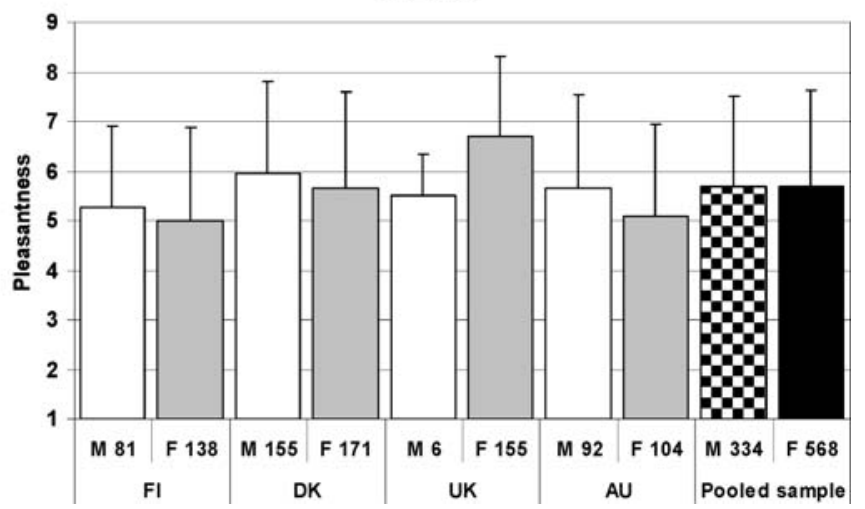

sample. The intensity scale were anchored with "No odor" (rating 1) and "Extremely strong odor" (rating 9) and the pleasantness scale with "Extremely unpleasant" (rating 1), "Neither pleasant nor unpleasant" (rating 5), and "Extremely pleasant" (rating 9). Pleasantness ratings of subjects who rated intensity as "No odor" were omitted

(common) environmental or non-additive genetic effects on the traits. Albeit large, the sample size may still have been inadequate to detect possible non-additive genetic effects.

A (negative) phenotypic correlation between intensity and pleasantness of androstenone may indicate that they have been influenced by an overlapping set of genes. To test this hypothesis, we performed the bivariate Cholesky decomposition of variance. We found a strong genetic correlation between the traits, suggesting an influence of the same or closely linked genes on the intensity and pleasantness of androstenone. As the environmental correlation was insignificant, the genetic correlation alone can be inferred to explain the whole phenotypic correlation between intensity and pleasantness.

Our results are in line with reports demonstrating that women are more sensitive to androstenone than are men (Wysocki and Gilbert 1989; Pierce et al. 2004). Present results are also consistent with earlier findings showing that children are more sensitive to androstenone than adults (Dorries et al. 1989; Wysocki and Gilbert 1989). The 


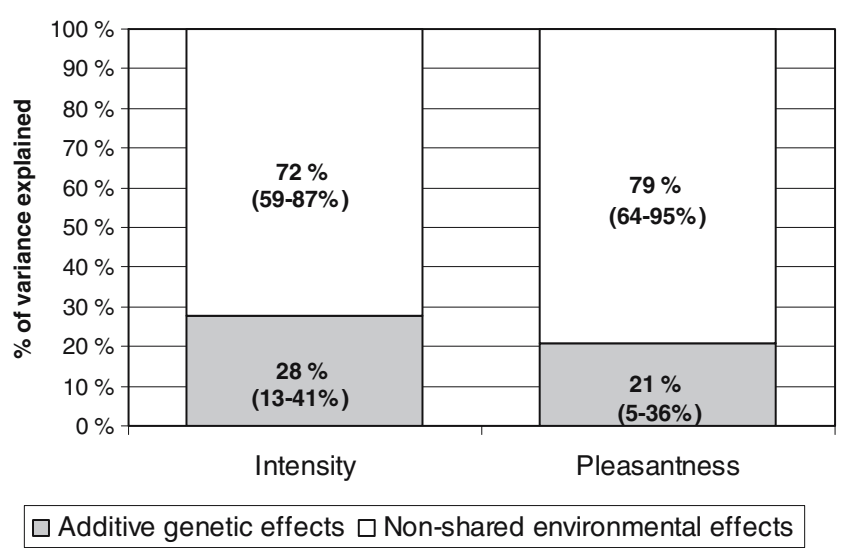

Fig. 4 Parameter estimates (95\% confidence intervals) of additive genetic and non-shared environmental effects for perceived intensity (all subjects, $n=907$ ) and pleasantness (subjects who rated intensity as 1, "No odor," omitted, remaining $n=728$ ) of androstenone odor. If apparently androstenone anosmic subjects (with intensity ratings 1 or 2) were omitted from both analyses (remaining $n=593$ ), additive genetic effects accounted for $28 \%(9-45 \%)$ and $23 \%(5-40 \%)$ of variance in intensity and pleasantness ratings, respectively

intensity of androstenone stimulus should not be too strong when it is used for investigating the olfactory perceptions because androstenone in high concentrations stimulates the trigeminal system (Boyle et al. 2006). Based on the distribution of the intensity ratings, the androstenone stimulus used in the present study was of appropriate intensity level.

The significant genetic component of variance in androstenone ratings raises a question about its evolutionary meaning. Androstenone is clearly an important determinant of behavior for some animals, but its pheromonal function in humans is unclear and controversial (Pause
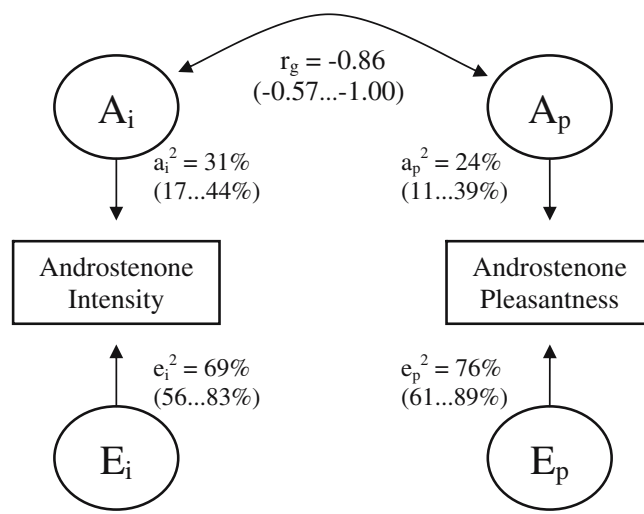

Fig. 5 The bivariate Cholesky decomposition of variance in perceived intensity and pleasantness of androstenone into additive genetic $(A)$ and non-shared environmental $(E)$ components, and their parameter estimates $\left(a^{2}, e^{2}\right)$. Environmental correlation between the traits was insignificant and omitted from the model, suggesting that genetic correlation $\left(r_{\mathrm{g}}\right)$ alone can explain the phenotypic correlation. The traits had $74 \%$ of genetic variance in common $\left(r_{\mathrm{g}}{ }^{2}\right)$. The $95 \%$ confidence intervals in parentheses
2004). Androstenone acts as a pheromone for pigs (Dorries et al. 1995) and is possibly involved in olfactory communication in non-human primates as well (Laska et al. 2005). As higher levels of androstenone occur in the axillary secretions of men than those of women, androstenone may serve as a signal for potential mates, as it does among pigs (Dalton et al. 2002). Although androstenone is a sex pheromone in pigs, it may have a different function in humans, and its efficiency may depend on other substances and contextual effects (Pause 2004).

In humans, the ability to detect androstenone may be an evolutionary relic. Humans appear to have lost part of their ability to detect androstenone during evolution, as humans are less sensitive to androstenone than mice and non-human primates (Laska et al. 2005). The ability to detect androstenone has probably not given selective advantage to the modern human species. Laska et al. (2005) found that androstenone anosmia is more frequent in humans than in non-human primates and suggested that this is due to a smaller number of functional odorant receptors in humans than in non-human primates. Evolution may have made androstenone useless in human mating and led to a higher prevalence of androstenone anosmia in humans compared with non-human primates.

To act as a pheromone, androstenone does not need to be detected as an odorous compound because pheromones do not necessarily have a distinct smell. Thus, the exposuredriven sensitization to androstenone (Wysocki et al. 1989) may be unrelated to its potential pheromonal function. Many animals respond to pheromonal signals without any prior experience or exposure (Stowers and Marton 2005). In other species, two or more substances often act synergistically to form a pheromonal signal. Therefore, the effect of androstenone may also depend on other substances emitted from humans (Pause 2004). However, exposure to a single structurally similar steroid occurring in male sweat, androstadienone, was recently reported to alter levels of the hormone cortisol in human female (Wyart et al. 2007).

\section{Conclusions}

Our results suggest that both the perceived intensity and pleasantness of androstenone odor are under moderate genetic control. Strong genetic correlation between the intensity and pleasantness implies that both traits are modified by the same or related genes that are responsible for phenotypic correlation between the traits. The results underline the importance of genetic influence on odor perceptions and motivates further search for underlying genes. 
Acknowledgments Quest International (presently Givaudan) is thanked for sponsoring the scratch-and-sniff odor stimuli. We thank the twins for their participation in the study, as well as Jytte Duerlund, Birgit Wich, Ann Eldridge, Marlene Grace, Daniel Park, Kaisu Taskila, Johanna Kuumola, Kati Kristiansson, Mari Lehtonen, Mari Siltala, Eero Vuoksimaa, Ursula Perks, and the TRU staff for their contribution to data collection. Kevin Deegan is thanked for his helpful suggestions on language of the manuscript. The Academy of Finland [grants 206327 (HT, AK, KK) and 108297 (KS)] and the European Commission [GenomEUtwin project under the 'Quality of Life and Management of the Living Resources' of 5th Framework Programme (QLG2-CT-2002-01254)] are acknowledged for funding. Data collection of Finnish twins was supported by the National Institutes of Health grants AA-12502 (to RJ Rose), and by the Academy of Finland grant 205585 (JK). JK, KS, and MP are supported by the Academy of Finland Centre of Excellence in Complex Disease Genetics.

\section{References}

Boulkroune N, Wang L, March A, Walker N, Jacob TJC (2007) Repetitive olfactory exposure to the biologically significant steroid androstadienone causes a hedonic shift and gender dimorphic changes in olfactory-evoked potentials. Neuropsychopharmacology 32(8): 1822-1829

Boyle JA, Lundström JN, Knecht M, Jones-Gotman M, Schaal B, Hummel T (2006) On the trigeminal percept of Androstenone and its implications on the rate of specific anosmia. J Neurobiol 66(13):1501-1510

Bremner EA, Mainland JD, Khan RM, Sobel N (2003) The prevalence of androstenone anosmia. Chem Senses 28(5):423-432

Dalton P, Doolittle N, Breslin PAS (2002) Gender-specific induction of enhanced sensitivity to odors. Nat Neurosci 5(3):199-200

Dorries KM, Schmidt HJ, Beauchamp GK, Wysocki CJ (1989) Changes in sensitivity to the odor of androstenone during adolescence. Dev Psychobiol 225(4):23-435

Dorries KM, Adkins-Regan E, Halpern BP (1995) Olfactory sensitivity to the pheromone, androstenone, is sexually dimorphic in the pig. Physiol Behav 57(2):255-259

Gross-Isseroff R, Ophir D, Bartana A, Voet H, Lancet D (1992) Evidence for genetic determination in human twins of olfactory thresholds for a standard odorant. Neurosci Lett 141(1):115-118

Kaprio J, Pulkkinen L, Rose R (2002) Genetic and environmental factors in health-related behaviors: studies on Finnish twins and twin families. Twin Res 5(5):366-371

Kaprio J (2006) Twin studies in Finland 2006. Twin Res Hum Genet 9 (6):772-777
Keller A, Zhuang H, Chi Q, Vosshall LB, Matsunami H (2007) Genetic variation in a human odorant receptor alters odour perception. Nature 449:468-472

Knaapila A, Keskitalo K, Kallela M, Wessman M, Sammalisto S, Hiekkalinna T, Palotie A, Peltonen L, Tuorila H, Perola M (2007) Genetic component of identification, intensity and pleasantness of odours: a Finnish family study. Eur J Hum Genet 15(5):596602

Labows JN, Wysocki CJ (1984) Individual differences in odor perception. Perfum Flavor 9(1):21-22, 24-26

Laska M, Wieser A, Salazar LTH (2005) Olfactory responsiveness to two odorous steroids in three species of nonhuman primates. Chem Senses 30(6):505-511

Neale MC, Cardon LR (1992) Methodology for Genetic Studies of Twins and Families. Kluwer, Dordrecht, The Netherlands

Neale MC, Boker SM, Xie G, Maes HH (2003) Mx: statistical modeling, 6th ednVCU, Department of Psychiatry. Richmond, VA, USA

Pause BM, Ferstl R, Fehm-Wolfsdorf G (1998) Personality and olfactory sensitivity. J Res Pers 32(4):510-518

Pause BM (2004) Are androgen steroids acting as pheromones in humans? Physiol Behav 83(1):21-29

Pierce JD, Cohen AB, Ulrich PM (2004) Responsivity to two odorants, androstenone and amyl acetate, and the affective impact of odors on interpersonal relationships. J Comp Psychol 118(1):14-19

Skytthe A, Kyvik K, Holm NV, Vaupel JW, Christensen K (2002) The Danish twin registry: 127 birth cohorts of twins. Twin Res 5 (5):352-357

Skytthe A, Kyvik K, Bathum L, Holm N, Vaupel JW, Christensen K (2006) The Danish twin registry in the new millennium. Twin Res Hum Genet 9(6):763-771

Spector TD, Williams MK (2006) The UK Adult Twin Registry (TwinsUK). Twin Res Hum Genet 9(6):899-906

Stowers L, Marton TF (2005) What is pheromone? Mammalian pheromones reconsidered. Neuron 46(5):699-702

Wright MJ, Martin NG (2004) Brisbane adolescent twin study: outline of study methods and research projects. Aust J Psychol. 56 (2):65-78

Wyart C, Webster WW, Chen JH, Wilson SR, McClary A, Khan RM, Sobel N (2007) Smelling a single component of male sweat alters levels of cortisol in women. J Neurosci 27(6):1261-1265

Wysocki CJ, Beauchamp GK (1984) Ability to smell androstenone is genetically determined. Proc Natl Acad Sci U S A 81(15):48994902

Wysocki CJ, Gilbert AN (1989) National Geographic smell surveyEffects of age are heterogenous. Ann NY Acad Sci 561:12-28

Wysocki CJ, Dorries KM, Beauchamp GK (1989) Ability to perceive androstenone can be acquired by ostensibly anosmic people. Proc Natl Acad Sci U S A 86(20):7976-7978 